\title{
Detection of multiple annexin autoantibodies in a patient with recurrent miscarriages, fulminant stroke and seronegative antiphospholipid syndrome
}

\author{
Philipp Scholz ${ }^{1}$, Markus Auler ${ }^{2,3}$, Bent Brachvogel ${ }^{2,3}$, Thomas Benzing ${ }^{4}$, Peter Mallman ${ }^{5}$, Thomas Streichert ${ }^{1}$, Andreas R. Klatt ${ }^{* 1}$ \\ ${ }^{1}$ Institute for Clinical Chemistry, University of Cologne, Germany \\ 2Department of Pediatrics and Adolescent Medicine, Experimental Neonatology, University of Cologne, Cologne, Germany \\ ${ }^{3}$ Center for Biochemistry, Medical Faculty, University of Cologne, Germany \\ ${ }^{4}$ Department II of Internal Medicine, University of Cologne, Cologne, Germany \\ ${ }^{5}$ Department of Obstetrics and Gynecology, University of Cologne, Cologne, Germany \\ *Corresponding author: andreas.klatt@uk-koeln.de
}

\begin{abstract}
Anti-phospholipid syndrome (APS) is one of the main causes for recurrent miscarriages. The diagnosis of APS is based on the occurrence of clinical symptoms such as thrombotic events or obstetric complications as well as the detection of antiphospholipid antibodies directed against $\beta 2$ glycoprotein I and cardiolipin, or a positive lupus anticoagulant assay. However, there is a subpopulation of patients with clinical symptoms of APS, but the lack of serological markers (seronegative APS). In addition, a large proportion of patients with unexplained recurrent miscarriages exist. These cases may be attributed, at least in part, to a seronegative APS.

The presence of autoantibodies against annexins is potentially associated with APS. Here we used immunoassays and immunoblots to detect autoantibodies directed against annexin A1-5, and A8, respectively, in a patient with a seronegative APS and a history of six recurrent pregnancy losses and fulminant stroke. We found strong $\lg M$ isotype antibody reactivity directed against annexin $\mathrm{A} 2$ and annexin $\mathrm{A} 8$, and moderate to weak $\lg \mathrm{M}$ isotype antibody reactivity directed against annexin $\mathrm{A1}, \mathrm{A} 3$, and $\mathrm{A} 5$. Further studies will evaluate the diagnostic value of IgM isotype antibodies against annexin A1-A5, and A8 for seronegative APS and recurrent miscarriages.
\end{abstract}

Key words: annexin; anti-phospholipid syndrome; anti-phospholipid antibody syndrome; seronegative anti-phospholipid syndrome; recurrent miscarriage

\section{Introduction}

Anti-phospholipid syndrome (APS) is an autoimmune disorder that leads to a hypercoagulative state. Clinically this disorder is characterized by any thrombotic event and/or specific obstetric complications, for instance preterm delivery and recurrent miscarriages (1-3). Therefore, APS is one of the main causes for pregnancy failure. Besides clinical symptoms, the presence of persistent circulating antiphospholipid antibodies (aPL) is used for the diagnosis of APS. Antiphospholipid antibodies are a heterogeneous family of autoantibodies against proteins binding to negatively charged phospholipids. They exert thrombogenic effects as they interfere with plasmatic components of the coagulation cascade, promote platelet aggregation and induce a pro-inflammatory and pro-coagulant endothelial phenotype (4). The relevant diagnostic aPL are IgG or IgM isotype autoantibodies directed against $\beta 2$-glycoprotein I (anti- $\beta 2 \mathrm{GPI}$ ) and cardiolipin (aCL), usually combined with the lupus anticoagulant (LA) functional assay. Patients with a "triple positivity" are thought to be at higher risk than those with single or double positivity to develop vascular thrombosis, pregnancy morbidity, and recurrent events $(5,6)$. The lupus anticoagulant test is the most predictive 
value for venous and arterial thrombosis in patients with suspected APS (7), whereas a high intermethod variability for aCL assays exists. This explains the low utility of aCL testing (8). The diagnostic value of the major epitope of B2GPI is still controversial discussed due to potential conformational changes during the immunoassay which may lead to epitope masking effects. Hence, there is no universal aPL- detection method (9). At present the diagnosis of an APS requires the detection of at least one of the three aPL, i.e. IgG or lgM isotype autoantibodies directed against $\beta 2$ glycoprotein I (anti- $\beta 2 \mathrm{GPI}$ ) and cardiolipin $(\mathrm{aCL})$, or a positive lupus anticoagulant (LA) functional assay. In addition, the revised 'Sapporo' criteria provide important details about the titres ( $>40 \mathrm{GPL}$ or MPL or $>99^{\text {th }}$ percentile for $\mathrm{aCL}$ and $>99^{\text {th }}$ percentile anti- $\beta 2 \mathrm{GPI}$ ) of aPL and their persistence in time (presence on two or more occasions at least 12 weeks apart) to reduce the probability of misdiagnosing APS in patients with thrombosis or pregnancy morbidity with transient or low titre aPL antibodies (5). Moreover there is an emerging group of autoantibodies potentially associated with APS (10). These autoantibodies are directed against proteins involved in coagulation, or cell membrane binding, but their clinical utility and diagnostic value remain unclear. This leads to a diagnostic gap in patients with clinical symptoms of an APS, but without evidence of established serological markers (seronegative APS, SNAPS), and may have fatal consequences for the patients.

Annexins (Anx) are a family of 12 highly conserved proteins characterized by their ability to bind phospholipids in a calcium dependent manner. Autoantibodies against annexins have been described in different diseases and, by now, autoantibodies against AnxA1, AnxA2, AnxA4, AnxA5 and AnxA11 have been detected in the serum of humans $(11,12)$. Recently, it was shown that AnxA5 self-assembles into 2D-arrays on membranes upon $\mathrm{Ca}^{2+}$ activation, and it has been suggested that AnxA5 competes with coagulation factors for phosphatidylserine binding to inhibit the activation of the coagulation cascade (13). This annexin is strongly expressed in the placenta (14) and by binding to negatively charged phospholipids at cell membranes (15) may be needed to maintain the placental integrity (16). Autoantibodies to AnxA5 have been proposed to be associated with the clinical features of APS, including thrombosis and recurrent miscarriages, and various research groups suggest autoantibodies against AnxA5 as additional serological criteria for APS (17).

In this study, we investigated the presence of $\lg G$ and IgM isotype autoantibodies directed against AnxA1-A5, and AnxA8 in a patient with a history of six pregnancy losses and a fulminant stroke, but with no evidence of the established diagnostic antibodies against $\beta 2 \mathrm{GPI}$ and $\mathrm{aCL}$, or $\mathrm{LA}$.

\section{Materials and methods}

\section{Subject}

At the age of 30, the female patient was diagnosed with systemic lupus erythematodes (SLE). Clinically the patient showed symptoms of pleurisy and of a lupus nephritis. In addition, elevated levels of anti-nuclear antibodies (ANA) and anti-double stranded DNA antibodies (dsDNA) were detected. The autoimmune disorder was managed with 125 $\mathrm{mg}$ cyclosporine and $5 \mathrm{mg}$ prednisolone. At the age of 34, the patient uttered the wish for pregnancy, but the patient suffered two early miscarriages. An evaluation of intrauterine abnormalities, transvaginal ultrasound scans and hysteroscopy gave no pathological changes. After the second miscarriage, the patient was furthermore tested for factor V-Leiden and prothrombin mutation, antithrombin, protein $C$ and protein $S$ deficiency, pathological factor VIII and factor XII activity, for anti-paternal antibodies, and several times for $\lg G$ and $\lg \mathrm{M}$ isotype autoantibodies against $\mathrm{aCL}$, and $L A$. None of these investigations gave pathological results. The detection assay for anti- $\beta 2 \mathrm{GPI}$ autoantibodies was not established at this time in our laboratory. Due to the clinical presentation, the patient has been diagnosed with seronegative APS. Therefore, an anticoagulative therapy with heparin was started after the second miscarriage, but the patient suffered three more early miscarriages. The gestational age of the miscarriages ranged from the $5^{\text {th }}$ to $8^{\text {th }}$ week of pregnancy. Af- 
ter the fifth miscarriage, the immunosuppressive therapy was changed to $5 \mathrm{mg}$ prednisolone every second day, and the patient received an anticoagulative and antiplatelet therapy with heparin and acetylsalicylic acid $100 \mathrm{mg} /$ day. The patient had another miscarriage, but also delivered a healthy child at the age of 38 and of 41 , respectively. As the patient was only clinical diagnosed with APS, the anticoagulative and antiplatelet medication was stopped after the second child birth. At the age of 42 , the patient suffered a fulminant stroke and presented a hemiparesis, hypoesthesia as well as an aphasia. Investigations gave no evidence of an embolic source, such as cardiac arrhythmia, cerebral vasculitis or actual autoimmune activity of the SLE.

\section{Blood samples}

Two blood samples (leftover samples) were taken into $4.7 \mathrm{~mL}$ serum monovettes (Sarstedt, Nümbrecht, Germany) and centrifuged at $3000 \times \mathrm{g}$ for 10 minutes, and analysed by immunoblot and immunoassay. The first sample was taken immediately after stroke; the second sample was picked 20 weeks later.

\section{Recombinant expression and purification of AnxA1-A5 and AnxA8}

Recombinant annexins were expressed in bacteria (BL21) and purified by affinity chromatography using glutathione Sepharose 4B (GE Healthcare, Uppsala, Sweden). The glutathione S-transferasetag was removed by cleavage with Factor Xa according to the manufacturer's protocol (Merck, Darmstadt, Germany). Purified annexins were dialyzed against 20 mM Hepes, 100 mM NaCl, pH 7.4. Endotoxin Removal Gel (Thermo Scientific, Rockford, USA) containing immobilized polymixin B was used in a column format to affinity-purify the annexins as described previously (15).

Sodium dodecyl sulfate-polyacrylamide gel electrophoresis (SDS-PAGE) and immunoblot - $1 \mu \mathrm{g}$ of recombinant annexin $\mathrm{A} 1-\mathrm{A} 5$, or $\mathrm{A} 8$, respectively, was separated on a $4-12 \%$ Bis-Tris gel (NuPAGE, Carlsbad, USA). For immunoblotting, the proteins were transferred to a polyvinylidene fluoride
(PVDF) membrane (0.45 $\mu \mathrm{m}$, Invitrogen, Carslbad, USA). Membranes were blocked in Tris buffered saline (TBS) with $0.05 \%$ Tween and 5\% milk powder, incubated with patient's serum 1:100 in TBS with $0.05 \%$ Tween and 5\% milk powder, and finally incubated and with horseradish peroxidase (HRP)conjugated goat anti-human IgG or anti-human IgM isotype antibodies (Invitrogen, Camarillo, USA) in TBS with $0.05 \%$ Tween and 5\% milk powder. Membranes were treated with electrochemiluminescence according to the manufacturer's instructions (GE Healthcare, Little Chalfont, UK), and bands detected with specific antibodies were visualized on Hyperfilm (GE Healthcare, Little Chalfont, UK).

Densitometric analyses of immunoblots were performed using ImageJ software. Therefore, pixel values were transformed into relative signal intensities of selected areas (18).

\section{Enzyme-linked immunosorbent assay (ELISA)}

Relative annexin autoantibody concentrations were determined by non-commercial ELISA. Therefore, $2 \mu \mathrm{g}$ recombinant annexin/well was coated overnight at $4{ }^{\circ} \mathrm{C}$ on a 96 -well plate (Nunc, Roskile, Denmark). The wells were subsequently incubated for 1 hour with the patient's serum (1:50) and HRP-conjugated goat anti-human IgM isotype antiserum (Invitrogen, Camarillo, USA). Signals were visualized by incubation with tetramethylbenzidine (TMB) (Sigma-Aldrich, St-Louis, USA). Each ELISA was assayed in duplicate and performed three times. Albumin (Sigma, St. Louis, USA) was used as negative control. The optical density (OD) was measured at $450 \mathrm{~nm}$. The OD correlates with antibody concentrations or antibody reactivity's, respectively, and fold change of antibody concentration was compared to the lowest value or control. The signal of buffer only was defined as cut off value.

\section{Biochemistry tests}

Routine laboratory biochemistry tests (serum creatinine, C-reactive protein) were run on a Roche Cobas C702 automated clinical chemistry analyser (Roche Diagnostics, Mannheim, Germany) with 
original Roche reagents. C3- and C4- complement factors were determined on a BNProSpec analyser (Siemens Healthcare Diagnostics, Marburg, Germany) with original Siemens reagents. A blood count was performed on a Sysmex XE-5000 analyser (Sysmex Corporation, Kobe, Japan). The erythrocyte sedimentation rate was measured on the automated ESR systems Sediplus S 2000 with S-Sedivettes (Sarstedt, Nümbrecht, Germany). LA functional assay was performed on a BCS analyser with Dade Actin FS or FSL Activated PTT Reagent (Siemens Healthcare Diagnostics, Marburg, Germany). IgG and IgM isotype antibodies against B2GPI were determined with a Quanta B2-GPI IgG or IgM-ELISA (Inova Diagnostics, San Diego, USA) on a DSX-ELISA-System (Dynex technologies, Chantilly, USA). Antibodies against CL were measured with an anti-cardiolipin IgG- or IgM-ELISA on an Euroimmun-Analyser I (Euroimmun, Lübeck, Germany). Antinuclear antibodies (ANA) were detected using the indirect immunofluorescence method on HEp-2 cells with a commercially available diagnostic kit (Euroimmun, Lübeck, Germany) according to the manufacturer's instructions. Antidouble-stranded DNA (anti-dsDNA) lgG isotype antibodies in serum were measured with the antidsDNA-NcX-ELISA (IgG) on an Euroimmun-Analyser I (Euroimmun, Lübeck, Germany).

\section{Results}

LA functional assay and IgG and IgM isotype antibodies against B2GPI or CL were determined immediately after stroke. IgG and IgM isotype antibodies against $B 2 \mathrm{GPI}$ or CL were additionally tested 9 months later. aPL could not be detected and there was no laboratory evidence for APS before and after the clinical event. In addition, similar ANA and anti-dsDNA antibodies, and serum creatinine levels were detected before and after stroke. ANA titre level was 1:1000, anti-dsDNA antibodies were negative, and serum creatinine concentration was around $130 \mu \mathrm{mol} / \mathrm{L}$. C3- and C4-complement factor, CRP, leukocytes and platelets were in the normal range. Erythrocyte sedimentation rate was $30 \mathrm{~mm} /$ hour (normal: < $14 \mathrm{~mm} /$ hour). In addition, the patient's serum was investigated by im- munoblot for the presence of annexin autoantibodies. We could identify strong reactivity of IgM isotype autoantibodies directed against AnxA2 and AnxA8, medium to low reactivity of IgM isotype autoantibodies against $A n x A 1, A n x A 3$, and AnxA5, and no antibody reactivity against AnxA4 and albumin. Antibodies of $\lg G$ isotype only showed a slight reactivity against $A n \times A 1$ and AnxA5 (Figure 1). The relative signal intensity of the immunoblot was evaluated by ImageJ (Figure 3) (18).

Furthermore, we verified the results of the immunoblot by ELISA. In accordance to the results of the immunoblot, we found high and persistent IgM isotype antibody reactivity directed against AnxA2 and $A n \times A 8$, and medium lgM isotype antibody reactivity directed against $A n \times A 1$ and AnxA3 (Figure 2).

\section{Discussion}

Here, we report the case of a woman diagnosed with seronegative APS and a history of six pregnancy losses and fulminant stroke, with no evidence of the diagnostic relevant aPL antibodies, but with persisting high IgM isotype autoantibodies reactivity against $A n x A 2$ and AnxA8.

As mentioned above, there is a growing number of aPL that may be involved in thrombosis or pregnancy morbidity, but only the three best investigated aPLs ( $\mathrm{aCL}$, a $2 \mathrm{GPI}$, and $\mathrm{LA})$ were included in the laboratory criteria of an APS. This leads to a diagnostic gap in patients with clinical symptoms of an APS, but without evidence of established serological markers. Since APS is a treatable cause of recurrent miscarriages, it is important to improve the identification of patients with a seronegative APS. Recently, autoantibodies against AnxA5 were found to be present in up to $17 \%$ of obstetric manifestations of APS cases, being the only serologic parameter significantly associated with miscarriage (19). However, due to inconsistent results among studies (8) the clinical correlation of autoantibodies against AnxA5 is still controversial discussed. Here, we detected moderate reactivity of IgM isotype antibodies against AnxA5, but we 

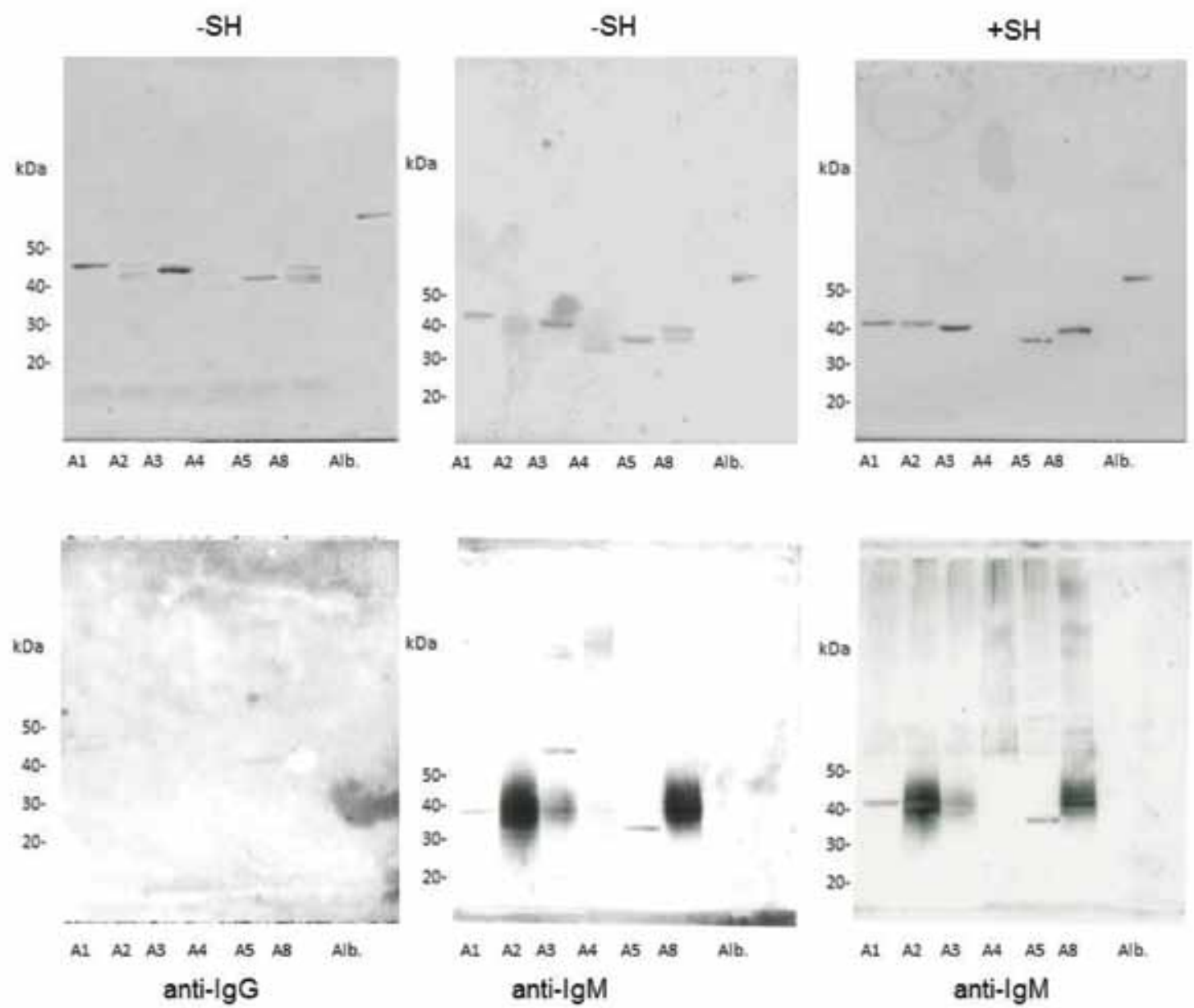

Figure 1. Immunoblot.

Serum of the patient was analysed immediately after stroke by Sodium dodecyl sulphate polyacrylamide gel electrophoresis following ponceau staining (upper row) and immunoblot (lower row) under reducing (+SH) and non-reducing conditions (-SH) for lgM and IgG isotype autoantibodies directed against annexin A1-A5 (A1-A5), and annexin A8 (A8). We found high lgM isotype autoantibody reactivity directed against annexin $A 2$ and annexin $A 8$, and almost no lgG isotype autoantibody reactivity against annexins. Albumin (Alb.) was used as negative control. kDa - kilo Dalton.

found considerably high reactivity of IgM isotype autoantibodies against AnxA2 and AnxA8 in the serum of our seronegative APS patient that suffered six miscarriages.

It was shown that AnxA2 interacts with $\beta 2 \mathrm{GPI}$ and Toll-like receptor 4 (TLR4) in a signalling complex leading to an inflammatory and pro-coagulant phenotype on endothelial cells $(20,21)$. Autoantibodies against AnxA2 were detected in APS patients, but the diagnostic sensitivity of the AnxA2 test is quite low, and AnxA2 autoantibodies were also detected in patients suffering from other autoimmune conditions lowering also the diagnostic specificity (8). AnxA8 is one of the least character- ized members of the annexin family. AnxA8 was described as an inhibitor of phospholipase $A 2$ and as a blood coagulation factor (22). As yet, there are no studies that have investigated AnxA8 antibodies in autoimmune conditions or other diseases. Here, we could show for the first time the presence of IgM isotype autoantibodies against AnxA2 and AnxA8 in a patient with seronegative APS.

There are various studies that investigate autoantibodies against annexin in the course of APS, for example autoantibodies against AnxA5 or AnxA2, but no study has investigated the presence of different annexin autoantibodies in a patient with APS. Furthermore, most of the investigations focus 


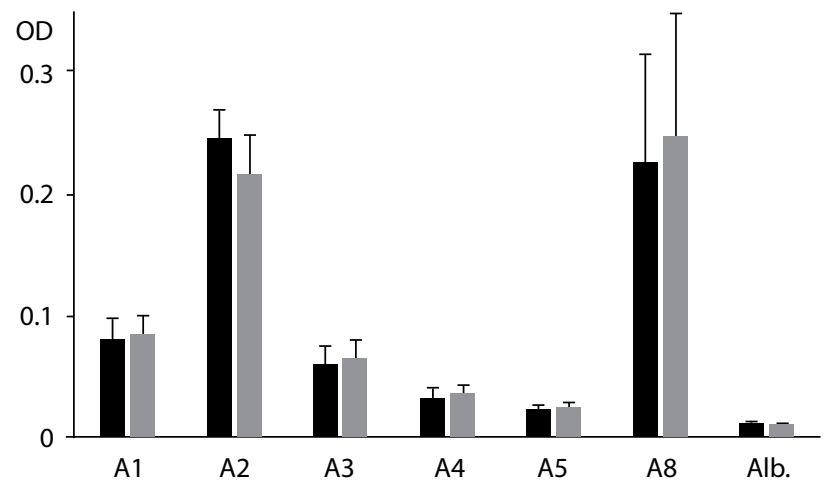

Figure 2. Anti-annexin enzyme-linked immunosorbent assay (ELISA).

Serum of the patient was analysed by ELISA for IgM isotype autoantibodies directed against annexin A1-A5 (A1-A5), and annexin A8 (A8), immediately after stroke (dark columns) as well as 20 weeks apart (light columns). We found high IgM isotype autoantibody reactivity directed against annexin $\mathrm{A} 2$ and annexin $A 8$, and medium IgM isotype autoantibody reactivity directed against annexin A1 and annexin A3. Each ELISA was assayed in duplicate and performed three times. The results are depicted as optical density (OD) values at $450 \mathrm{~nm}$. Error bars represents the standard deviation. Albumin (Alb.) was used as negative control.

on IgG isotype autoantibodies. Three studies investigated associations between circulating lgM isotype autoantibodies against AnxA5 and obstetric complications and interestingly in one study, IgM isotype autoantibodies against AnxA5 were found to be stronger associated with obstetric complications than IgG isotype autoantibodies (23). In this patient, we found high IgM isotype antibody reactivity directed against Anx2 and Anx8.

IgM isotype antibodies appear early in the course of an infection and there are various studies reporting infect associated transient autoantibodies $(24,25)$. Infect associated autoantibodies are the reason why repeated serological analysis of aPL within 12 weeks are mandatory in the diagnostic criteria of APS. In this patient, we investigated the persistence of the circulating annexin antibodies and could demonstrate persisting IgM isotype autoantibodies against $\mathrm{An} \times \mathrm{A} 2$ and AnxA8 in constant titres over a period of 20 weeks.

In the last years, different technical approaches for the detection of aPL have been developed. These

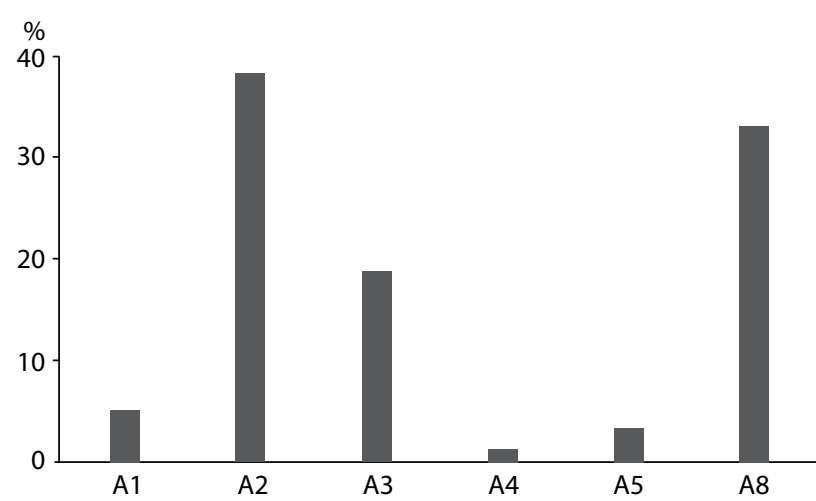

Figure 3. Transformation of pixel values into relative signal intensities (in percent, \%) of the anti-IgM immunoblot (+SH) with Image J.

The relative signal intensities of annexin A1-A5 (A1-A5), and annexin A8 (A8) were calculated using Image J software. Comparison of the relative signal intensities of the immunoblot and the enzyme-linked immunosorbent assay yielded consistent results, with strongest IgM isotype autoantibody reactivity directed against annexin $\mathrm{A} 2$ and annexin $\mathrm{A} 8$.

techniques comprise solid phase immunoassays (e.g. ELISA), multiline dot assays (dot blot) and thin layer chromatography (8). Most of the studies were performed with solid phase assays, e.g. ELISAs. Of course, ELISAs allow the quantification of the corresponding antibody, but lacks of specificity. To overcome this restriction, we used both, ELISA as well as immunoblot analysis, to verify antibodies against AnxA2 and AnxA8 in the patient's serum. In this patient, we therefore can exclude unspecific cross-reactivity of the detected annexin autoantibodies.

In conclusion, we describe for the first time the presence of autoantibodies directed against different annexins and persistent lgM isotype autoantibody reactivity directed against AnxA2 and AnxA8 in a patient with seronegative APS who suffered six miscarriages. Further studies in additional patients are needed to evaluate and proof that the detection of different annexin autoantibodies elevate the diagnostic sensitivity and specificity to bridge the diagnostic gap for patients with seronegative APS and unexplained recurrent miscarriages.

\section{Potential conflict of interest}

None declared. 


\section{References}

1. Cervera R, Piette JC, Font J, Khamashta MA, Shoenfeld $Y$, Camps MT, et al. Antiphospholipid syndrome: clinical and immunologic manifestations and patterns of disease expression in a cohort of 1,000 patients. Arthritis Rheum 2002;46:1019-27. http://dx.doi.org/10.1002/art.10187.

2. Cervera R, Khamashta MA, Shoenfeld Y, Camps MT, Jacobsen $S$, Kiss $E$, et al. Morbidity and mortality in the antiphospholipid syndrome during a 5-year period: a multicentre prospective study of 1000 patients. Ann Rheum Dis 2009;68:1428-32. http://dx.doi.org/10.1136/ard.2008.093179.

3. Miyakis S, Lockshin MD, Atsumi T, Branch DW, Brey RL, Cervera $R$, et al. International consensus statement on an update of the classification criteria for definite antiphospholipid syndrome (APS). J Thromb Haemost 2006;4:295-306. http:// dx.doi.org/10.1111/j.1538-7836.2006.01753.x.

4. Meroni PL, Borghi MO, Raschi E, Tedesco F. Pathogenesis of antiphospholipid syndrome: understanding the antibodies. Nat Rev Rheumatol 2011;7:330-9. http://dx.doi. org/10.1038/nrrheum.2011.52.

5. Galli M. The antiphospholipid triangle. J Thromb Haemost 2010;8:234-6. http://dx.doi.org/10.1111/j.15387836.2009.03689.x.

6. Pengo V, Ruffatti A, Legnani $C$, Gresele P, Barcellona D, Erba $N$, et al. Clinical course of high-risk patients diagnosed with antiphospholipid syndrome. J Thromb Haemost 2010;8:23742. http://dx.doi.org/10.1111/j.1538-7836.2009.03674.x.

7. Devreese KM. Antiphospholipid antibodies: evaluation of the thrombotic risk. Thromb Res 2012;130:S37-40. http:// dx.doi.org/10.1016/j.thromres.2012.08.270.

8. Misasi R, Capozzi A, Longo A, Recalchi S, Lococo E, Alessandri $C$, et al. "New" antigenic targets and methodological approaches for refining laboratory diagnosis of antiphospholipid syndrome. J Immunol Res 2015;2015:858542. http:// dx.doi.org/10.1155/2015/858542.

9. Chighizola CB, Gerosa M, Meroni PL. New tests to detect antiphospholipid antibodies: anti-domain I beta-2-glycoprotein-I antibodies. Curr Rheumatol Rep 2014;16:402. http:// dx.doi.org/10.1007/s11926-013-0402-7.

10. Forastiero $R$, Martinuzzo M. The emerging role of multiple antiphospholipid antibodies positivity in patients with antiphospholipid syndrome. Expert Rev Clin Immunol 2015;11:1255-63. http://dx.doi.org/10.1586/174466 6X.2015.1080121.

11. laccarino L, Ghirardello A, Canova M, Zen M, Bettio S, Nalotto $L$, et al. Anti-annexins autoantibodies: their role as biomarkers of autoimmune diseases. Autoimmun Rev 2011;10:5538. $h$ ttp://dx.doi.org/10.1016/j.autrev.2011.04.007.

12. Satoh A, Suzuki K, Takayama E, Kojima K, Hidaka T, Kawakami $M$, et al. Detection of anti-annexin IV and $V$ antibodies in patients with antiphospholipid syndrome and systemic lupus erythematosus. J Rheumatol 1999;26:1715-20.

13. Bouter A, Gounou C, Berat R, Tan S, Gallois B, Granier T, et al. Annexin- $A 5$ assembled into two-dimensional arrays promotes cell membrane repair. Nat Commun 2011;2:270. http://dx.doi.org/10.1038/ncomms 1270 .
14. Brachvogel B, Moch $H$, Pausch F, Schlotzer-Schrehardt $U$, Hofmann C, Hallmann $R$, et al. Perivascular cells expressing annexin A5 define a novel mesenchymal stem cell-like population with the capacity to differentiate into multiple mesenchymal lineages. Development 2005;132:2657-68. http://dx.doi.org/10.1242/dev.01846.

15. Rosenbaum S, Kreft S, Etich J, Frie C, Stermann J, Grskovic $l$, et al. Identification of novel binding partners (annexins) for the cell death signal phosphatidylserine and definition of their recognition motif. J Biol Chem 2011;286:5708-16. http://dx.doi.org/10.1074/jbc.M110.193086.

16. Ueki $H$, Mizushina T, Laoharatchatathanin T, Terashima $R$, Nishimura $Y$, Rieanrakwong $D$, et al. Loss of maternal annexin $A 5$ increases the likelihood of placental platelet thrombosis and foetal loss. Sci Rep 2012;2:827. http:// dx.doi.org/10.1038/srep00827.

17. Bertolaccini ML, Amengual O, Atsumi T, Binder WL, de Laat $B$, Forastiero $R$, et al. 'Non-criteria' aPL tests: report of a task force and preconference workshop at the 13th International Congress on Antiphospholipid Antibodies, Galveston, TX, USA, April 2010. Lupus 2011;20:191-205. http://dx.doi. org/10.1177/0961203310397082.

18. Rasband WS. ImageJ, U. S. National Institutes of Health, Bethesda, Maryland, USA, http://imagej.nih.gov/ij/, 1997-2015.

19. Bizzaro N, Tonutti E, Villalta D, Tampoia M, Tozzoli R. Prevalence and clinical correlation of anti-phospholipid-binding protein antibodies in anticardiolipin-negative patients with systemic lupus erythematosus and women with unexplained recurrent miscarriages. Arch Pathol Lab Med 2005; 129:61-8.

20. Romay-Penabad Z, Montiel-Manzano MG, Shilagard T, Papalardo $E$, Vargas $G$, Deora $A B$, et al. Annexin $A 2$ is involved in antiphospholipid antibody-mediated pathogenic effects in vitro and in vivo. Blood 2009;114:3074-83. http://dx.doi. org/10.1182/blood-2008-11-188698.

21. Cańas F, Simonin L, Couturaud F, Renaudineau Y. Annexin A2 autoantibodies in thrombosis and autoimmu ne diseases. Thromb Res 2015;135:226-30. http://dx.doi. org/10.1016/j.thromres.2014.11.034.

22. Hauptmann R, Maurer-Fogy I, Krystek E, Bodo G, Andree $H$, Reutelingsperger CP. Vascular anticoagulant beta: a novel human $\mathrm{Ca} 2+/$ phospholipid binding protein that inhibits coagulation and phospholipase A2 activity. Its molecular cloning, expression and comparison with VACalpha. Eur J Biochem 1989;185:63-71. http://dx.doi. org/10.1111/j.1432-1033.1989.tb15082.x.

23. Sater MS, Finan RR, Mustafa FE, Al-Khateeb GM, Almawi WY. Anti-annexin V IgM and IgG autoantibodies and the risk of idiopathic recurrent spontaneous miscarriage. J Reprod Immunol 2011;89:78-83. http://dx.doi.org/10.1016/j. jri.2010.12.009.

24. Avcin T, Toplak N. Antiphospholipid antibodies in response to infection. Curr Rheumatol Rep 2007;9:212-8. http:// dx.doi.org/10.1007/s11926-007-0034-x.

25. von Landenberg P, Doring Y, Modrow S, Lackner KJ. Are antiphospholipid antibodies an essential requirement for an effective immune response to infections? Ann $N Y$ Acad Sci 2007;1108:578-83. http://dx.doi.org/10.1196/ annals. 1422.060 . 